\title{
Entanglement of spin-orbit qubits induced by Coulomb interaction
}

\author{
Y. N. Fang ${ }^{1}$, Yusuf Turek ${ }^{1}$, J. Q. You ${ }^{2}$, and C. P. Sun ${ }^{1,2 *}$ \\ ${ }^{1}$ State Key Laboratory of Theoretical Physics, Institute of Theoretical Physics, \\ Chinese Academy of Sciences, and University of the Chinese Academy of Sciences, Beijing 100190, China \\ ${ }^{2}$ Beijing Computational Science Research Center, Beijing 100084, China
}

\begin{abstract}
Spin-orbit qubit (SOQ) is the dressed spin by the orbital degree of freedom through a strong spinorbit coupling. We show that Coulomb interaction between two electrons in quantum dots located separately in two nanowires can efficiently induce quantum entanglement between two SOQs. The physical mechanism to achieve such quantum entanglement is based on the feasibility of the SOQ responding to the external electric field via an intrinsic electric dipole spin resonance.
\end{abstract}

PACS numbers: 68.65.Hb, 71.70.Ej, 03.67.Bg

\section{INTRODUCTION}

Entanglement of particles now has been considered as quantum resource to implement protocols of quantum information processing [1]. To achieve quantum entanglement for various kinds of qubits is a central task in quantum information science and technology. With very strong spin-orbit coupling (SOC), an electron spin becomes the so called spin-orbit qubit (SOQ) [2], which has been experimentally implemented in quantum nanowires. Its bit states essentially are two dressed spin states incorporating with the orbital degree of freedom (DOF), and thus could feasibly respond to both electric and magnetic fields. In this sense quantum manipulations on SOQ can be achieved via electron-dipole spin resonance [4, 5].

With those existing progresses, we will face with a crucial task: how to efficiently achieve entanglement of two SOQs? To this end we refer to our previous investigations to create inter-spin entanglement through SOC incorporating with Coulomb interaction [7. In quantum electrodynamics (QED), the static Coulomb interaction is a consequence of virtual photon exchange between two electric charges, thus can be regraded as an external electric field exerted by one electric charge on another. This implies that the SOQs can also feasibly respond to the Coulomb interaction and thus cause a correlated motion of two SOQs for generating quantum entanglement between SOQs. In ref. [7, a scheme was proposed to create entanglement between two local spins (rather than the SOQs) in two 2D quantum dots mediated with SOC.

In this paper, we study an alternative scheme to generate entanglement between two SOQs through Coulomb interaction. We will show that this Coulomb interaction mediated entanglement can be optimized by the strength of SOC: it is not the case the stronger the SOC is, higher the entanglement is. More specifically, we consider a low dimensional system made up by two electrons separately confined in two paralleled nanowires and subjected to a weakly external magnetic field. By treating the magnetic

*Electronic address: cpsun@csrc.ac.cn field as perturbation and modeling the Coulomb interaction linearly nearby the equilibrium point, we derive an effective Hamiltonian for two SOQs, with two qubits flip-flop induced by the Coulomb interaction. It is found that the flip-flop rate as well as two qubits entanglement (concurrence) changes non-monotonically with respect to SOC strength, and they can be optimized by SOC. This discovery largely modifies the intuitionistic observation that stronger coupling is more helpful in creating highly entangled many body states.

This paper is organized as follow, in Sec. II we model the Coulomb interaction as a linear coupling for two electrons trapped in two quantum dots which are located in two parallel nanowires. In Sec. III, we elucidate subspaces that can be used to encode SOQ under a limiting situation where magnetic field can be treated as perturbation to orbital motion as well as SOC. In Sec. IV, we discuss the generation of the correlated flip-flop processes of two qubits as mediated by the Coulomb interaction, and derive the effective Hamiltonian for SOQs encoded using nearly degenerated ground states. Then we study entanglement in the two SOQs system by calculating system dynamics as well as time evolution of concurrence.

\section{MODEL SETUP FOR TWO PARALLEL NANOWIRES}

We consider two parallel 1D nanowires placed along $\mathrm{x}$-axis in the $\mathrm{x}-\mathrm{z}$ plane. Two electrons are confined by biased electrodes aside each nanowire [3], as schematically plotted in Fig. 1. By treating the confining potentials as harmonic traps, we can write down the Hamiltonian for the two electrons with SOCs

$$
H=\sum_{j=1,2}\left[\frac{p_{j}^{2}}{2 m}+V_{j}+\alpha_{j} \sigma_{j}^{x} p_{j}+\mu_{j} \sigma_{j}^{z}\right]+V_{c},
$$

where $j=1,2$ indicates confined electron in the front or at the rear; $m$ is electron effective mass; $p_{j}$ and $x_{j}$ are momentum and coordinate for $\mathrm{j}$-th electron. We take the external trap to be of harmonic oscillator, i.e., $V_{j}=$ $m \bar{\omega}_{j}^{2} x_{j}^{2} / 2$, and $\bar{\omega}_{j}$ is characteristic oscillator frequency of the trap; $\alpha_{j}$ is the Dresselhaus SOC strength and $2 \mu_{j}$ is 


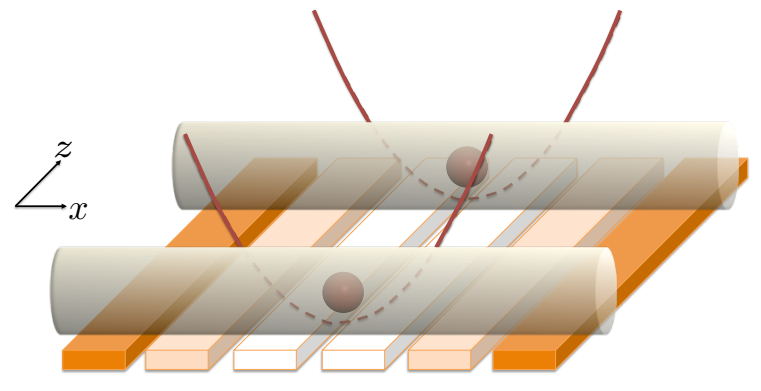

Figure 1: Schematic of two electrons confined in quantum dots which are located separately in two 1D parallel nanowires. Electrodes beside nanowires provide harmonic traps for electrons. Those trapping potentials are represented by red parabolic curves.

the Zeeman splitting for $\mathrm{j}$-th electron [6]. $V_{c}$ is Coulomb interaction between two electrons

$$
V_{c}=\frac{e^{2}}{4 \pi \varepsilon_{0} \varepsilon_{r} \sqrt{\left(x_{1}-x_{2}\right)^{2}+\left(z_{1}-z_{2}\right)^{2}}} .
$$

Let $l_{j}=\sqrt{\hbar / m \bar{\omega}_{j}}$ be the characteristic length of harmonic trap and $z_{0}$ be the separation between two nanowires. In the case of strong confinement, i.e, $l_{j} \ll$ $z_{0}, V_{c}$ can be further simplified [7. Specifically, we replace $z_{1}-z_{2}$ in $V_{c}$ by $z_{0}$ and expand Eq. (2) around $x_{1}-x_{2}=0$. Then the potential is written up to second order of $x_{1}-x_{2}$ as

$$
V_{c} \approx V_{0}-\frac{1}{2} m \omega_{c}^{2}\left(x_{1}-x_{2}\right)^{2},
$$

where $V_{0}=e^{2} /\left(4 \pi \varepsilon_{0} \varepsilon_{r} z_{0}\right)$ and $\omega_{c}=\sqrt{V_{0} /\left(m z_{0}^{2}\right)}$.

With above approximation, the system reduces to two coupled harmonic oscillators with SOC, with the Hamiltonian

$H=\sum_{j=1,2}\left[\frac{p_{j}^{2}}{2 m}+\frac{1}{2} m \omega_{j}^{2} x_{j}^{2}+\alpha_{j} \sigma_{j}^{x} p_{j}+\mu_{j} \sigma_{j}^{z}\right]+m \omega_{c}^{2} x_{1} x_{2}$.

Here, we have ignored the constant term $V_{0}$ and defined $\omega_{j}=\sqrt{\bar{\omega}_{j}^{2}-\omega_{c}^{2}}$. We remark here that $\bar{\omega}_{j}>\omega_{c}$ always hold under the pre-assumed condition $l_{j} \ll z_{0}$, thus $\omega_{j}$ is a physically meaningful parameter.

In terms of the bosonic creation and annihilation operators $a_{j}^{\dagger}$ and $a_{j}, p_{j}$ and $x_{j}$ are rewritten as $p_{j}=i \sqrt{\hbar \omega_{j} m / 2}\left(a_{j}^{\dagger}-a_{j}\right)$ and $x_{j}=\sqrt{\hbar /\left(2 m \omega_{j}\right)}\left(a_{j}^{\dagger}+\right.$ $\left.a_{j}\right)$, respectively. The Hamiltonian becomes $H=$ $\sum_{j=1,2}\left(H_{j, 0}+H_{j, 1}\right)+V$, where

$$
\begin{gathered}
H_{j, 0}=\hbar \omega_{j}\left(a_{j}^{\dagger} a_{j}+\frac{1}{2}\right)+i \xi_{j}\left(a_{j}^{\dagger}-a_{j}\right) \sigma_{j}^{x} \\
V=g\left(a_{1}^{\dagger}+a_{1}\right)\left(a_{2}^{\dagger}+a_{2}\right) .
\end{gathered}
$$

and $H_{j, 1}=\mu_{j} \sigma_{j}^{z}$. In the above equations, $\xi_{j}=$ $\sqrt{m \hbar \omega_{j} / 2} \alpha_{j}$ are the rescaled SOC strength and we have introduced the coupling constant $g=\hbar \omega_{c}^{2} /\left(2 \sqrt{\omega_{1} \omega_{2}}\right)$. (a)

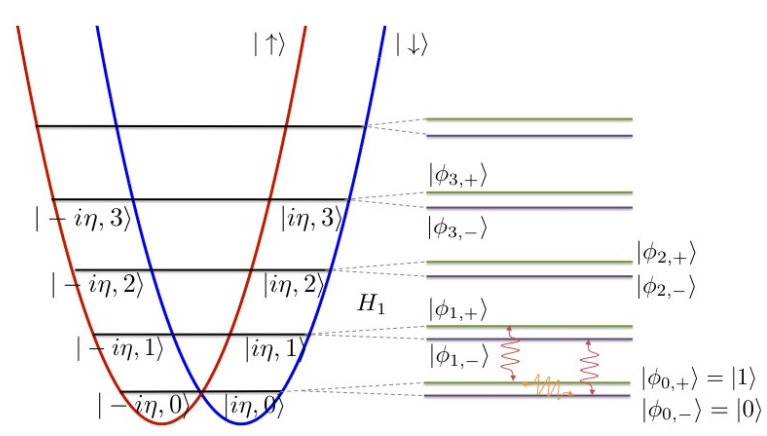

(b)

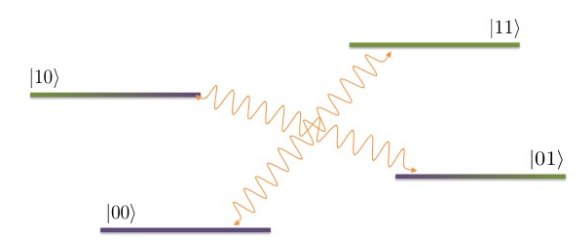

Figure 2: (a) Energy level structure of a spin with SOC in a weak magnetic field: two parabolas indicate different harmonic traps felt by electron with spin states $|\uparrow\rangle$ or $|\downarrow\rangle$. Black lines inside parabolas denote eigenstates of $H_{0}$, which are the spin dependent displaced Fock states. When $H_{1}$ is introduced, each two degenerated levels split as shown on the right. A SOQ can be encoded into the nearly degenerate ground states $\left|\phi_{0, \pm}\right\rangle$. Transition between two qubit's basis can be achieved via external a.c. electronic driving, which is shown by wavy arrows. The off-resonance (red arrows) can be ignored under resonant driving. (b) Two SOQs coupled by the linearized Coulomb interaction, the Coulomb interaction here is of the same role as the a.c. field in the single qubit case. However, only transitions between two pairs of basis are allowed due to generalized parity selection rules.

\section{DRESSED SPIN QUBIT IN MAGNETIC FIELD}

The existence of SOC in $H_{j, 0}$ couples spatial motion of each electron to its internal spin DOF. Therefore, it provides us with an active method to manipulate spin states of electrons via controlling over their spatial DOF [9]. In this section, we study the driven SOC system in the limit where $\mu_{j} \ll \hbar \omega_{j}$ and $\mu_{j} \ll \xi_{j}$, i.e., the strong SOC dominates over the Zeeman effect.

First, let us analyze the energy level configuration of $H_{j, 0}$. The two fold degenerated eigenvectors of $H_{j, 0}$ with the eigenvalue $E_{j, n}=\hbar \omega_{j}\left(n-\eta_{j}^{2}+1 / 2\right)$ are $\mid n, \uparrow$ \rangle$_{j}=\left|-i \eta_{j}, n\right\rangle_{j}|\uparrow\rangle_{j}$ and $|n, \downarrow\rangle_{j}=\left|i \eta_{j}, n\right\rangle_{j}|\downarrow\rangle_{j}$. Here, $|\alpha, n\rangle_{j}=D_{j}(\alpha)|n\rangle_{j}$ is displaced Fock state created by displaced operator $D_{j}(\alpha)$ for $a_{j}$ and $a_{j}^{\dagger}\left[8 ; ; \eta_{j}=\xi_{j} /\left(\hbar \omega_{j}\right)\right.$ is reduced SOC strength; $|\uparrow(\downarrow)\rangle_{j}$ are eigenstates of $\sigma_{j}^{x}$ with eigenvalues \pm 1 . The following analysis are identical for both electrons, thus we omit sub-index $\mathrm{j}$ for the clarity of presentation in rest of this section.

As shown in Fig. 2a, since $H_{0}$ and $H_{1}$ do not commute with each other, thus applying a magnetic field will then 
flip electron spin and lift the previously degenerated levels. Generally the level splitting will be proportional to the strength of the applied field in weak field limit, so the two split levels are nearly degenerate and consequently they can be used to encode a qubit, which is known as SOQ.

In order to find out the level splitting as well as wave functions for the dressed spin, we apply degenerate state perturbation theory on $H_{1}$ by treating $\epsilon=\mu /(\hbar \omega)$ as a small parameter. Following the procedure given in ref. [10], we first combine two degenerate eigenstates of $H_{0}$ such that $H_{1}$ is diagonal within their subspace. Since $\sigma^{z}$ flips $|\uparrow\rangle$ to $|\downarrow\rangle$, the diagonal matrix elements $\left\langle n, s\left|H_{1}\right| n, s\right\rangle$ vanish, where $s$ stands for $\uparrow$ or $\downarrow$, and offdiagonal elements are

$$
\left\langle n, s\left|H_{1}\right| n,-s\right\rangle=\mu e^{-2 \eta^{2}} L_{n}\left(4 \eta^{2}\right) .
$$

Here, $L_{n}(x)$ is Laguerre polynomials [11].

In the two dimensional subspace spanned by $\{\mid n, \uparrow$ \rangle$,|n, \downarrow\rangle\}, H_{1}$ is then written as

$$
H_{1}=\mu e^{-2 \eta^{2}} L_{n}\left(4 \eta^{2}\right)(|n, \uparrow\rangle\langle n, \downarrow|+h . c .),
$$

The orthonormal eigenstates of above two-by-two matrix are $|n, \pm\rangle=\frac{1}{\sqrt{2}}(|n, \uparrow\rangle \pm|n, \downarrow\rangle)$, the corresponding eigenvalues are

$$
\delta E_{n, \pm}= \pm \mu e^{-2 \eta^{2}} L_{n}\left(4 \eta^{2}\right) .
$$

From the zero-th order wave function $|n, \pm\rangle$, we can use the perturbation method to obtain the eigenvalue of $H_{0}+$ $H_{1}$ as

$$
E_{n, \pm}=E_{n}+\delta E_{n, \pm}
$$

where the first order correction $\delta E_{n, \pm}$ is given by Eq. (9). The corresponding first order eigenfunctions $\left|\phi_{n, \pm}^{(1)}\right\rangle$ are calculated in Appendix A. Two states with lowest energies $E_{0, \mp}$ are denoted by $|0\rangle \equiv\left|\phi_{0,-}^{(1)}\right\rangle$ and $|1\rangle \equiv\left|\phi_{0,+}^{(1)}\right\rangle$, respectively. They are written explicitly as

$$
\begin{aligned}
|N\rangle= & \left|0,(-1)^{N-1}\right\rangle+(-1)^{N} \sum_{m>0}\left[\lambda_{2 m-1}\left|2 m-1,(-1)^{N}\right\rangle\right. \\
& \left.+\lambda_{2 m}\left|2 m,(-1)^{N-1}\right\rangle\right], \quad(N=0,1)
\end{aligned}
$$

where $\lambda_{m}=\epsilon\langle m \mid 2 i \eta\rangle / m$.

Energy splitting between $|1\rangle$ and $|0\rangle$ is given by $\Delta \equiv$ $\delta E_{0,+}-\delta E_{0,-}=2 \mu \exp \left(-2 \eta^{2}\right) \ll \hbar \omega$ since $\epsilon \ll 1$. $\hbar \omega$ is level spacing between two nearest unperturbed levels. If all relevant interactions are approximately in resonant with $|0\rangle$ and $|1\rangle$, then the two states can be used to encode quantum information as a SOQ. Aside from this, it worths to remark another property of the SOQ. We notice that $|n, \pm\rangle$ looks similar to even and odd coherent states [13, which process definite parities when the arguments of displacement operator are purely imaginary. Here, as the direction of displacement in $|n, \pm\rangle$ is entangled with spin orientation, they are eigenstates of following generalized parity (GP) operator

$$
\Lambda=\Pi \sigma^{z},
$$

where $\Pi$ is the usual parity operator defined by $\Pi^{2}=1$ and $\Pi|x\rangle=|-x\rangle[15] . p, x$ and $\sigma^{x}$ are odd under $\Lambda$, which means $\{\Lambda, p\}=0$ etc., and $\Lambda$ commutes with $H_{0}+H_{1}$. Therefore, SOQ basis $|0\rangle$ and $|1\rangle$ can be simultaneously eigenstates of $\Lambda$, i.e., $\Lambda|0\rangle=-|0\rangle$ and $\Lambda|1\rangle=|1\rangle$. Furthermore, as shown in Appendix B, $\left|\phi_{n,+}^{(1)}\right\rangle$ and $\left|\phi_{n+1,-}^{(1)}\right\rangle$ have the GPs that are different from $\left|\phi_{n,-}^{(1)}\right\rangle$ and $\left|\phi_{n+1,+}^{(1)}\right\rangle$.

This property of $\left|\phi_{n, \pm}^{(1)}\right\rangle$ then indicates following selection rules. First, for $\mathrm{H}_{1}$, following transitions are forbidden

$$
\left|\phi_{n, \pm}^{(1)}\right\rangle \leftrightarrow\left|\phi_{n+2 m-1, \pm}^{(1)}\right\rangle,\left|\phi_{n, \pm}^{(1)}\right\rangle \leftrightarrow\left|\phi_{n+2 m, \mp}^{(1)}\right\rangle
$$

since $H_{1}$ is even under $\Lambda$, i.e., $\left[H_{1}, \Lambda\right]=0$. Second, for the parity odd operator $a^{\dagger}+a \propto x$, following transitions are forbidden

$$
\left|\phi_{n, \pm}^{(1)}\right\rangle \leftrightarrow\left|\phi_{n+2 m, \pm}^{(1)}\right\rangle,\left|\phi_{n, \pm}^{(1)}\right\rangle \leftrightarrow\left|\phi_{n+2 m-1, \mp}^{(1)}\right\rangle
$$

\section{ENTANGLEMENT BETWEEN TWO SOQS}

After elucidating the level structure and defining SOQ basis in previous section, now we study how Coulomb interaction between two electrons can be used to facilitate entanglement between SOQs. To this end, we calculate the matrix elements of the linearized Coulomb interaction Eq. (6) in the basis of two SOQs subspace spanned by $\{|00\rangle,|01\rangle,|10\rangle,|11\rangle\}$, where $|M N\rangle=|M\rangle_{1} \otimes|N\rangle_{2}$.

As shown in Appendix C, up to second order in $\epsilon_{j}$, matrix elements of the Coulomb interaction are given by

$$
\begin{aligned}
& \left\langle N_{1} N_{2}|V| M_{1} M_{2}\right\rangle \\
= & (-1)^{N_{1}+\bar{N}_{2}} \delta_{N_{1}, \bar{M}_{1}} \delta_{N_{2}, \bar{M}_{2}} J,
\end{aligned}
$$

where $\bar{N}=0(1)$ if $N=1(0)$, and the two qubits effective flip-flop strength $J$ is obtained as

$$
J=16 g \epsilon_{1} \epsilon_{2} \eta_{1} \eta_{2} e^{-2\left(\eta_{1}^{2}+\eta_{2}^{2}\right)} .
$$

Existence of exponential decay term in $J$ indicates a non-monotone behavior with respect to SOC. In fact, as shown in Fig. 3a, by setting $\eta_{j}=1 / \sqrt{2}$ the flip-flop strength is optimized to $J_{o p t}=8 g \epsilon_{1} \epsilon_{2} \exp (-2)$.

The possible transitions caused by the Coulomb interaction are illustrated in Fig. 2b, where only transitions $|00\rangle \leftrightarrow|11\rangle$ and $|01\rangle \leftrightarrow|10\rangle$ are allowed. To illustrate why others are forbidden, we recall a definition of the parity operator as $\Pi_{j}=\exp \left(i \pi a_{j}^{\dagger} a_{j}\right)$ [16]. Using this definition, $a_{j}$ and $a_{j}^{\dagger}$ can be shown to be odd under the GP operation $\Lambda$. According to selection rules similar to Eq.(14), GP of $|0\rangle$ and $|1\rangle$ must change after emitting or absorbing an phonon. On the other hand, at first order 


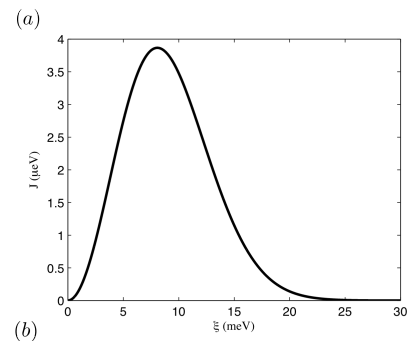

(b)

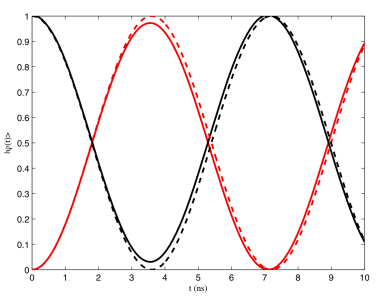

$(c)$

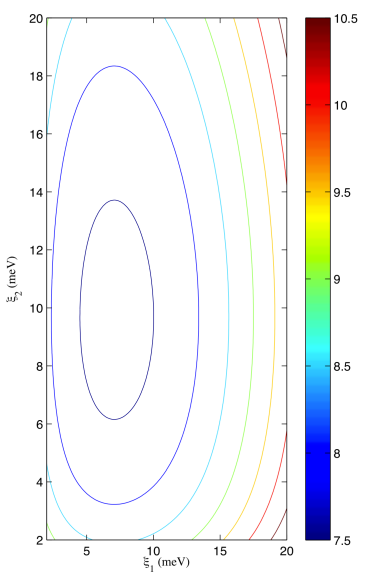

Figure 3: (a) Coulomb interaction induced SOQ effective flip-flop strength $J$ as a function of rescaled SOC strength $\xi_{1}=\xi_{2}=\xi$. (b) Time evolution of two SOQs' wave function $|\psi(t)\rangle$ under resonant condition $\Delta_{1}=\Delta_{2}$ and $|\psi(0)\rangle=|10\rangle$. Red and black lines are $|\langle 01 \mid \psi(t)\rangle|^{2}$ and $|\langle 10 \mid \psi(t)\rangle|^{2}$, respectively. Solid lines are calculated from Eq.(17), while dashed lines are exact result from $H$. (c) Minimal time $T$ needed to complete a two qubits basis flip in resonant case as a function of the rescaled SOC strength $\xi_{1}$ and $\xi_{2}$. The plot is logarithm rescaled in $\mathrm{z}$ direction to stress the existence of minimal point. Except for (c), parameters used in calculation are: $\bar{\omega}_{1}=20 \mathrm{meV}, \bar{\omega}_{2}=15 \mathrm{meV}, \omega_{c}=5 \mathrm{meV}, \mu_{1}=\mu_{2}=1 \mathrm{meV}$ and $\eta_{1}=\eta_{2}=0.8$.

the linearized Coulomb interaction results in exchanging of an phonon between two electrons. Therefore, the GP of each SOQ states must change as a result of the interaction. Therefore, all transitions with only one SOQ changes its GP such as $|00\rangle \leftrightarrow|01\rangle$ will vanish.

Introducing the following pseudo-spin operators $S_{j,+}=$ $|1\rangle_{j j}\langle 0|, S_{j,-}=S_{j,+}^{\dagger}$ and $S_{j, z}=\left[S_{j,+}, S_{j,-}\right]$, the Hamiltonian of the two SOQ subsystem within the subspace of the two qubits is written as

$$
\begin{aligned}
H_{e f f}= & \sum_{j=1,2} \Delta_{j} S_{j, z}-J\left(S_{1,+} S_{2,+}-S_{1,+} S_{2,-}\right. \\
& + \text { h.c. }) .
\end{aligned}
$$

Here, we have ignored a constant energy shift $2\left(E_{1,0}+\right.$ $\left.E_{2,0}\right)$.

With qubits flip-flop processes, entanglement between two SOQs can be generated. To quantitatively discuss this, we study evolution dynamics of the two qubits system as well as their concurrence. For the two SOQs system initially in $|\psi(0)\rangle=|10\rangle$, the wave function at time $\mathrm{t}$ is given by

$$
|\psi(t)\rangle=(\cos \Omega t+i \sin \theta \sin \Omega t)|10\rangle+i \cos \theta \sin \Omega t|01\rangle
$$

Here, $\Omega=\sqrt{J^{2}+\left(\Delta_{1}-\Delta_{2}\right)^{2}}$ and $\theta=\arctan \left[\left(\Delta_{1}-\right.\right.$ $\left.\left.\Delta_{2}\right) / J\right]$ are Rabi frequency and mixing angle, respectively.
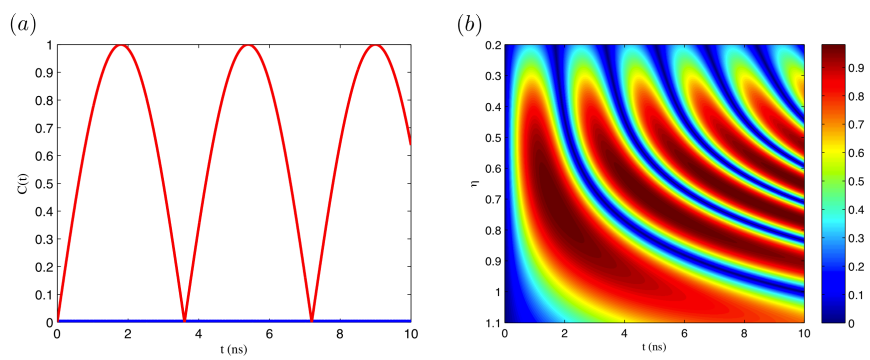

Figure 4: (a) Time evolution of concurrence $C(t)$ for two SOQs under initial states $|10\rangle$ or $|01\rangle$ (red) and initial states $|00\rangle$ or $|11\rangle$ (blue) in resonant case, where maximum entangled states can be generated at specific time. (b) Concurrence $C(t)$ for initial state $|10\rangle$ or $|01\rangle$ as functions of time and reduced SOC strength $\eta_{1}=\eta_{2}=\eta$ for nearly resonant case. Except $\mu_{2}=1.01 \mathrm{meV}$ for calculating (b), parameters used in calculating those plots are the same as Fig. 3a,b.

Time evolution of $|\psi(t)\rangle$ is shown in Fig. 3b under resonant condition $\Delta_{1}=\Delta_{2}$, which requires a homogenous magnetic field over two nanowires. In this case $|10\rangle$ and $|01\rangle$ are degenerated in energy. Since $\theta=0$, thus the two degenerated basis flip completely. Time needed for a complete flip $T=2 \pi / J$ is plotted as function of $\xi_{j}$ and shown in Fig. 3c. Clearly we see that by adjusting SOC, we can achieve the shortest operation time.

We also calculated time evolution of concurrence $C(t)$ for two SOQs to quantify degree of entanglement among them, using a formula for two qubits in pure state [12], for initial state $|\psi(0)\rangle=|10\rangle$ or $|01\rangle$

$$
C(t)=|\sin \Omega t| \sqrt{\sin ^{2} 2 \theta \sin ^{2} \Omega t+4 \cos ^{2} \theta \cos ^{2} \Omega t} .
$$

Notice that $C(t)=C(t+\pi / \Omega)$. When $\tan ^{2} \theta \leq 1, C(t)$ always have two maximum values at

$$
t_{ \pm}^{*}=\frac{1}{\Omega} \arccos \left[ \pm \sqrt{\frac{1}{2}\left(1-\tan ^{2} \theta\right)}\right]
$$

for $\Omega t \in[0, \pi]$. The corresponding maximum values are equal and given by $C\left(t_{ \pm}^{*}\right)=1$. On the other hand, if $\tan ^{2} \theta>1$, then $C(t)$ has only one maximum at $t^{*}=$ $\pi /(2 \Omega)$ for $\Omega t \in[0, \pi]$, and the value is $C\left(t^{*}\right)=|\sin 2 \theta|$.

While for the rest two initial states, i.e., $|11\rangle$ and $|00\rangle$, the concurrence $C^{\prime}(t)$ is also given by Eq. 19 except $\Omega$ and $\theta$ being replaced by

$$
\Omega^{\prime}=\sqrt{J^{2}+\left(\Delta_{1}+\Delta_{2}\right)^{2}}, \theta^{\prime}=\arctan \frac{\Delta_{1}+\Delta_{2}}{J} .
$$

Time evolution of $C(t)$ are shown in Fig. 4a and Fig. $4 \mathrm{~b}$, where we see that in resonant case the system can always evolve into a maximally entangled state. For nearly resonant case, maximum value of $C(t)$ depends non-monotonically on SOC and can be optimized by tuning SOC. However, it is hardly to observe any entanglement if initial state is chosen as $|11\rangle$ or $|00\rangle$. Because $\Delta_{1}+\Delta_{2} \gg J, \theta^{\prime}$ approaches $\pi / 2$ in this case, thus $C^{\prime}(t)$ becomes vanishingly small. 


\section{CONCLUSION}

In this paper, we have studied entanglement induced by the Coulomb interaction in a system of two electrons, which are separately trapped in two 1D nanowires with SOC. We explicitly shown that how the presence of magnetic field can enable the two electron spins to encode SOQs in a regime where SOC and orbital motion of electrons dominate over the Zeeman effect. The SOQ basis are shown to process the definite GP, thus lead to selection rules under a large set of external driving forces including the electronic field.

Based on the feasibly responding of SOQs to electric field [14], we have shown that two qubits flip-flop can be effectively created via the inter-electrons Coulomb interaction. In resonant case the flip between two SOQs is perfect and the its period is found to depend strongly on SOC and changing non-monotonically, which is in contrary to an intuitive thinking. By studying the time evolution of concurrence, we shown that entanglement among two SOQs in a nearly resonant case can be optimized by adjusting the strength of SOC.

Finally let us remark that it is possible to generate equally efficient entanglement between $|00\rangle$ and $|11\rangle$ just by reversing the direction of the external magnetic field on one SOQ setup, i.e., using inhomogenous magnetic fields. Since the two basis become energy-degenerated if the fields on both SOQ setups are of the same magnitude. Meanwhile, the coupling between all the rest pairs of basis can be achieved by single qubit operations.

\section{Acknowledgments}

This work is supported by National Natural Science Foundation of China under Grants No.11121403, No.10935010, No.11074261, No. 91121015 and and the National 973 program (Grant No. 2012CB922104 and No. 2014CB921402).

\section{Appendix A: SOQ wave functions by perturbation theory}

The first order eigenstates are calculated according to following formula 10 .

$$
\left|\phi_{n, \pm}^{(1)}\right\rangle=|n, \pm\rangle+\sum_{m \neq n} \sum_{s^{\prime}= \pm} \frac{\left\langle m, s^{\prime}\left|H_{1}\right| n, \pm\right\rangle}{E_{n}-E_{m}}\left|m, s^{\prime}\right\rangle,
$$

Matrix elements in the denominator can be rewritten as

$$
\left\langle m, \pm\left|H_{1}\right| n, \pm\right\rangle= \pm \mu\left\langle m\left|D(2 i \eta)+D^{\dagger}(2 i \eta)\right| n\right\rangle
$$

as well as

$$
\left\langle m, \mp\left|H_{1}\right| n, \pm\right\rangle= \pm \mu\left\langle m\left|D(2 i \eta)-D^{\dagger}(2 i \eta)\right| n\right\rangle .
$$

Displacement operator under Fock states can be expressed in terms of generalized Laguerre polynomials
$L_{n}^{(m)}(x)$ [11], thus above matrix elements can be rewritten as

$$
\left\langle m\left|D(2 i \eta) \pm D^{\dagger}(2 i \eta)\right| n\right\rangle=\frac{1}{\mu}\left(E_{n}-E_{m}\right) \kappa_{m, n}^{( \pm)}
$$

where $\kappa_{m, n}^{( \pm)}=\mu \zeta_{m, n}\left[1+(-1)^{|m-n|}\right] /\left(E_{n}-E_{m}\right)$ and

$$
\zeta_{m, n}=\frac{1}{2}\left(\frac{n !}{m !}\right)^{f(m-n)}(2 i \eta)^{|m-n|} L_{n}^{(|m-n|)}\left(4 \eta^{2}\right)
$$

with auxiliary function $f(x)=1 / 2$ if $x \geq 0$ and $-1 / 2$ otherwise.

Insert Eqs. A4 and A5 back to Eq. A1,

$$
\begin{aligned}
& \left|\phi_{n, \pm}^{(1)}\right\rangle \\
= & |n, \pm\rangle \pm \sum_{m \neq n}\left(\kappa_{m, n}^{(+)}|m, \pm\rangle+\kappa_{m, n}^{(-)}|m, \mp\rangle\right) .
\end{aligned}
$$

For two lowest states where $n=0$, coefficients $\kappa_{m, 0}^{( \pm)}$are rewritten as

$$
\kappa_{m, 0}^{( \pm)}=-\left[1 \pm(-1)^{m}\right] \lambda_{m}
$$

where $\lambda_{m}$ is defined in the main text. In this case, Eq. A6 recovers Eq. 111.

\section{Appendix B: GP of perturbed wave functions}

First notice that, for displaced Fock states

$$
\begin{aligned}
\Pi| \pm i \eta, 2 n\rangle & =\int d x|-x\rangle\langle x \mid \pm i \eta, 2 n\rangle \\
& =\int d x e^{ \pm i m \alpha x / \hbar} \varphi_{2 n}(x)|-x\rangle \\
& =\int d x e^{\mp i m \alpha x / \hbar} \varphi_{2 n}(x)|x\rangle \\
& =|\mp i \eta, 2 n\rangle,
\end{aligned}
$$

as well as

$$
\Pi| \pm i \eta, 2 n-1\rangle=-|\mp i \eta, 2 n-1\rangle,
$$

where $\varphi_{n}(x)$ is eigenstates of 1D harmonic oscillator.

When spin is included, following similar relations can be derived

$$
\begin{aligned}
\Lambda|2 n, \uparrow\rangle & =\Lambda|-i \eta, 2 n\rangle|\uparrow\rangle \\
& =|i \eta, 2 n\rangle|\downarrow\rangle \\
& =|2 n, \downarrow\rangle,
\end{aligned}
$$

as well as

$$
\begin{gathered}
\Lambda|2 n, \downarrow\rangle=|2 n, \uparrow\rangle, \Lambda|2 n-1, \uparrow\rangle=-|2 n-1, \downarrow\rangle \\
\Lambda|2 n-1, \downarrow\rangle=-|2 n-1, \uparrow\rangle
\end{gathered}
$$

Those relations then indicate that $|n, \pm\rangle$ are eigenstates of the GP operator $\Lambda$

$$
\begin{gathered}
\Lambda|2 n,+\rangle=|2 n,+\rangle, \Lambda|2 n-1,-\rangle=|2 n-1,-\rangle \\
\Lambda|2 n,-\rangle=-|2 n,-\rangle, \Lambda|2 n-1,+\rangle=-|2 n-1,+\rangle
\end{gathered}
$$


From Appendix A, $\kappa_{m, n}^{(+)}\left(\kappa_{m, n}^{(-)}\right)$is non-zero only if $m$ and $n$ have same (opposite) oddness. Therefore, Eq. A6 is rewritten as follow for $n$ even,

$$
\begin{aligned}
& \left|\phi_{2 n, \pm}^{(1)}\right\rangle \\
= & |2 n, \pm\rangle \pm \sum_{m \neq n}\left(\kappa_{2 m, 2 n}^{(+)}|2 m, \pm\rangle+\kappa_{2 m-1,2 n}^{(-)}|2 m-1, \mp\rangle\right),
\end{aligned}
$$

Together with Eq. B5, we then concluded that

$$
\Lambda\left|\phi_{2 n,+}^{(1)}\right\rangle=\left|\phi_{2 n,+}^{(1)}\right\rangle, \Lambda\left|\phi_{2 n,-}^{(1)}\right\rangle=-\left|\phi_{2 n,-}^{(1)}\right\rangle,
$$

and similarly analysis for the $n$ odd case gives

$$
\Lambda\left|\phi_{2 n-1,+}^{(1)}\right\rangle=-\left|\phi_{2 n-1,+}^{(1)}\right\rangle, \Lambda\left|\phi_{2 n-1,-}^{(1)}\right\rangle=\left|\phi_{2 n-1,-}^{(1)}\right\rangle .
$$

\section{Appendix C: Matrix elements of the Coulomb interaction}

In the subspace of two SOQs, matrix element of the linearized Coulomb interaction is written as

$$
\begin{aligned}
& \left\langle N_{1} N_{2}|V| M_{1} M_{2}\right\rangle \\
= & g\left\langle N_{1}\left|a_{1}^{\dagger}+a_{1}\right| M_{1}\right\rangle\left\langle N_{2}\left|a_{2}^{\dagger}+a_{2}\right| M_{2}\right\rangle .
\end{aligned}
$$

Since $a_{j}^{\dagger}+a_{j}$ is odd under GP operation with respect to j-th electron, thus $N_{j}$ and $M_{j}$ must be different in order to have non-zero matrix elements (since $|0\rangle$ and $|1\rangle$ have different GP as shown in the main text). Therefore,

$$
\begin{aligned}
& \left\langle N_{1} N_{2}|V| M_{1} M_{2}\right\rangle \\
= & \delta_{N_{1}, \bar{M}_{1}} \delta_{N_{2}, \bar{M}_{2}} J_{N_{1}, N_{2}},
\end{aligned}
$$

where $J_{m n}=g\left\langle m\left|a_{1}^{\dagger}+a_{1}\right| \bar{m}\right\rangle\left\langle n\left|a_{2}^{\dagger}+a_{2}\right| \bar{n}\right\rangle$.

Matrix element $\left\langle n\left|a^{\dagger}+a\right| \bar{n}\right\rangle$ can be calculated directly from Eq. 111. Up to first order in $\epsilon$, it is given by

$$
\left\langle n\left|a^{\dagger}+a\right| \bar{n}\right\rangle=-2 i(-1)^{n} \operatorname{Im} \lambda_{1},
$$

Thus $J_{m n}$ can be rewritten as

$$
\begin{aligned}
J_{m n} & =4(-1)^{m+n-1} g \prod_{j=1,2} \operatorname{Im} \epsilon_{j}\left\langle 1 \mid 2 i \eta_{j}\right\rangle \\
& =(-1)^{m+\bar{n}} 16 g \epsilon_{1} \epsilon_{2} \eta_{1} \eta_{2} e^{-2 \eta_{1}^{2}-2 \eta_{2}^{2}} .
\end{aligned}
$$

Then Eq. 15 can be recovered by inserting Eq. (C4) back to Eq. (C2). Notice that although $J_{m n}$ is second order in $\epsilon_{j}$, actually we do not need to do perturbation theory on wave function to that order. Because direct calculation shows $\left\langle 0, s\left|a^{\dagger}+a\right| 0, s^{\prime}\right\rangle=0$ even for $s \neq s^{\prime}$, then effect from the second order correction of wave function on matrix element of the Coulomb interaction must be higher order and thus can be neglected.
[1] Ryszard Horodecki, Paweł Horodecki, Michał Horodecki, Karol Horodecki, Rev. Mod. Phys. 81, 865-942 (2009).

[2] S. Nadj-Perge, S. M. Frolov, E. P. A. M. Bakkers, and L. P. Kouwenhoven, Nature 468, 1084-1087 (2010).

[3] Christian Flindt, Anders S. Sørensen, and Karsten Flensberg, Phys. Rev. Lett. 97, 240501 (2006).

[4] Rui Li, J. Q. You, C. P. Sun, and Franco Nori, arXiv:1304.3257.

[5] E. I. Rashba, and Al. L. Efros, Phys. Rev. Lett. 91, 126405 (2003).

[6] G. Dresselhaus, Phys. Rev. 100, 580 (1955).

[7] Nan Zhao, L. Zhong, Jia-Lin Zhu, and C. P. Sun, Phys. Rev. B 74, 075307 (2006).

[8] D.F. Walls and Gerard J. Milburn, Quantum Optics (Springer-Verlag, Berlin, Heidelberg, 1994).

[9] Christian Flindt, Anders S Sørensen and Karsten Flensberg, J. Phys.: Conf. Ser. 61, 302 (2007).
[10] J. J. Sakurai, Jim Napolitano, Modern Quantum Mechanics 2nd ed., (Addison-Wesley, 2011).

[11] A. Ferraro, S. Olivares, M. G. Paris, Gaussian States in Quantum Information, Napoli. Series on physics and astrophys. (2005).

[12] Charles H. Bennett, David P. DiVincenzo, John A. Smolin, and William K. Wootters, Phys. Rev. A 54, 3824 (1996).

[13] V.V. Dodonov, I.A. Malkin, V.I. Man'ko, Physica (Utrecht) 72, 597 (1974).

[14] K. C. Nowack, F. H. L. Koppens, Yu. V. Nazarov, L. M. K. Vandersypen, Science 318, 1430 (2007).

[15] Claude Cohen-Tannoudji, Bernard Diu, Franck Laloë, Quantum mechanics, (Wiley-VCH, 1992).

[16] Antonino Messina, and Gheorghe Draganescu, ArXiv: 1306. 2524. 